\title{
Characterizing Pediatric Lung Ultrasound Findings During a Chemically-induced Bronchospasm
}

\author{
Francois Gagnon ${ }^{1}$, Noah Marzook ${ }^{2}$, Alexandre Deragon ${ }^{2}$, Larry Lands $^{3}$, David Zielinski ${ }^{4}$, \\ Adam Shapiro $^{5}$, and Alexander S. Dubrovsky ${ }^{2}$ \\ ${ }^{1}$ University of Ottawa Department of Pediatrics \\ ${ }^{2}$ McGill University Faculty of Medicine \\ ${ }^{3}$ McGill University Health Centre \\ ${ }^{4}$ McGill University \\ ${ }^{5}$ Montreal Children's Hospital, McGill University
}

July 2, 2021

\begin{abstract}
Background: Lung ultrasound (LUS) has been shown to be a useful clinical tool in pediatrics. LUS has been well studied in other respiratory conditions, but very little is known about the LUS findings of asthma. Objectives: The primary objective was to characterize LUS findings of pediatric patients before and after a chemically-induced bronchospasm. The secondary objective was to evaluate the effect of bronchodilators on the LUS findings. Methods: Eligible patients 6 to 17 years old presenting for a methacholine challenge test (MCT) in a pediatric respiratory clinic were recruited. LUS was performed before and after the MCT as well as after bronchodilator administration. LUS were analysed by an expert blinded to the patient characteristics and MCT results. Results: 44 patients were included in the study. 5 patients had positive LUS findings at baseline. 15 patients had a positive LUS following the MCT. There was a significant association between having a chemically-induced bronchospasm and a positive LUS post-MCT ( $\mathrm{p}=0.05$, odds ratio 5.3, 95\% CI [1.0-27.7]). Among patients who developed positive LUS findings post-MCT, 4 out of 9 returned to having a negative LUS post-bronchodilator administration. Conclusions: This is the first known report of an association between LUS findings and bronchospasm in pediatric patients. It is also the first documentation of resolution of LUS findings post-bronchodilator administration. Most LUS findings observed were small and limited to one or a few intercostal spaces. Further research is required to evaluate the effect of bronchodilators on LUS in the emergency department.
\end{abstract}

\section{Characterizing Pediatric Lung Ultrasound Findings During a Chemically-induced Bron- chospasm}

Francois Gagnon ${ }^{\mathrm{a}} \mathrm{MD}$, Noah Marzook ${ }^{\mathrm{a}} \mathrm{MD}$, Alexandre Deragon ${ }^{\mathrm{b}}$ FRCPC, David Zielinski ${ }^{\mathrm{c}}$ FRCPC, Adam J. Shapiro ${ }^{c}$ FRCPC, Larry C. Lands ${ }^{c}$ FRCPC, Alexander S. Dubrovsky ${ }^{\mathrm{d}}$ FRCPC

${ }^{a}$ Division of General Pediatrics, Montreal Children's Hospital, McGill University Health Center, Montreal, Quebec, Canada

${ }^{b}$ Division of Pediatric Emergency Medicine, Children's Hospital of Eastern Ontario, Ottawa, Ontario, Canada

${ }^{c}$ Department of Respirology, Montreal Children's Hospital, McGill University Health Center, Montreal, Quebec, Canada

${ }^{d}$ Pediatric Emergency Medicine, Montreal Children's Hospital, McGill University Health Center, Montreal, Quebec, Canada 
Investigation site : Montreal Children's hospital, Respirology clinics, 1001 Décarie Blvd, Montreal, Qc, $\mathrm{Ca}, \mathrm{H} 4 \mathrm{~A} 3 \mathrm{~J} 1$

Meeting : This study was presented at the 2020 Pediatric Emergency Research Canada annual meeting (January 2020, Tremblant, Quebec, Canada).

Sources of funding and support : Funding for the research assistant was provided by an innovation grant from the Department of Pediatric of the Montreal Children's Hospital - McGill University Health Center. The principal investigator (ASD) are members of the Research Institute of the McGill University Health Center, which is supported by the Fonds de Recherche du Québec-Santé.

Contact : Francois Gagnon, 1725 Frobisher lane, apt 110, K1G 0E6, fgagnon@cheo.on.ca, 1-438-492-9520

Keywords : Asthma, Ultrasonography, Child, Bronchial Provocation Tests

Abbreviated Title : Pediatric Lung Ultrasound during a Bronchospasm

\section{Abstract}

Background: Lung ultrasound (LUS) has been shown to be a useful clinical tool in pediatrics. LUS has been well studied in other respiratory conditions, but very little is known about the LUS findings of asthma.

Objectives: The primary objective was to characterize LUS findings of pediatric patients before and after a chemically-induced bronchospasm. The secondary objective was to evaluate the effect of bronchodilators on the LUS findings.

Methods: Eligible patients 6 to 17 years old presenting for a methacholine challenge test (MCT) in a pediatric respiratory clinic were recruited. LUS was performed before and after the MCT as well as after bronchodilator administration. LUS were analysed by an expert blinded to the patient characteristics and MCT results.

Results: 44 patients were included in the study. 5 patients had positive LUS findings at baseline. 15 patients had a positive LUS following the MCT. There was a significant association between having a chemicallyinduced bronchospasm and a positive LUS post-MCT ( $\mathrm{p}=0.05$, odds ratio 5.3, 95\% CI [1.0-27.7]) . Among patients who developed positive LUS findings post-MCT, 4 out of 9 returned to having a negative LUS post-bronchodilator administration.

Conclusions: This is the first known report of an association between LUS findings and bronchospasm in pediatric patients. It is also the first documentation of resolution of LUS findings post-bronchodilator administration. Most LUS findings observed were small and limited to one or a few intercostal spaces. Further research is required to evaluate the effect of bronchodilators on LUS in the emergency department.

\section{Introduction}

\subsection{Background}

Asthma represents one of the most common chronic conditions of childhood ${ }^{16,25}$. It is a leading cause for both emergency department (ED) visits and hospital admissions amongst Canadian children ${ }^{30}$.

It is of primordial importance that emergency physicians be able to rapidly recognize respiratory distress and accurately formulate a working diagnosis which will guide initial stabilization \& therapeutic efforts. It can be difficult to discriminate acute asthma from pulmonary infection, pleural effusion, lobar atelectasis or pneumothorax. No serological or radiological signs allow a specific diagnosis of an acute asthma exacerbation. The diagnosis of this condition still relies on clinical features such as a thorough medical history and physical examination $^{3,30}$.

In his sentinel study, Lichtenstein ${ }^{24}$ showed that point-of-care lung ultrasound (LUS) immediately provided the diagnosis of acute respiratory failure in $90.5 \%$ of cases of adult patients with respiratory failure. A negative lung profile on ultrasound predicted the diagnosis of asthma, whereas a positive LUS identified pneumo- 
nia/empyema or pulmonary edema. Since that initial study, LUS has made its way to the pediatric emergency department (PED) ${ }^{15,17,23,24,27,34,40}$. LUS has been shown to be superior to other classical diagnostic imaging modalities in identifying common pediatric respiratory pathologies such as pneumonia ${ }^{1,5,9,11,13,19,20,32,34-36,39}$, bronchiolitis $^{2,4}$, pulmonary effusions $6^{6,9,10,20,22,29,35}$ and pneumothorax ${ }^{7,33}$.

Very little is known about LUS findings in pediatric asthma. Dankoff et al. ${ }^{12}$ studied LUS of known asthmatic children 2 to 17 years old presenting to the PED with an asthma exacerbation. Of the 60 children studied, $45 \%$ had positive ultrasound findings. In the sub-group 6 to 17 years old, in which the diagnosis of asthma is often more evident, $23 \%$ of patients had positive ultrasound findings. Positive ultrasound findings correlated with hospitalization and PED length of stay. It is unclear why such a large number of children with asthma exacerbation had LUS findings compared to what is suggested in the adult literature ${ }^{24}$. Numerous hypotheses were suggested to explain this discrepancy, but it is clear that further research is required in order to shed light on this surprising difference ${ }^{12}$.

A methacholine challenge test (MCT) is a recognized diagnostic test for asthma ${ }^{26}$. It consists of providing increasingly higher doses of methacholine to induce bronchospasm and evaluate for airway hyperresponsiveness ${ }^{8}$. MCTs offer the unique opportunity to study before and after induced bronchospasm. Studying LUS evolution of patients undergoing an MCT might provide some insights on the patho-physiologic relation between LUS findings in asthmatic children presenting to the emergency department with respiratory distress.

\subsection{Importance}

Despite asthma exacerbations being common, very little is known about LUS findings during pediatric bronchospasm. As point of care ultrasound use increases, it is important to define LUS findings in asthma exacerbation in children to help clinicians interpret them appropriately during diagnostic evaluations.

\subsection{Goals of this investigation}

The primary objective of this study was to characterize LUS findings in children presenting to a MCT at baseline and after chemically-induced bronchospasm. The secondary objective was to determine the effect of bronchodilators on LUS findings.

\section{Methods}

\subsection{Study design and setting}

This was a prospective cohort study conducted from December 2017 to June 2019 in the pulmonary function laboratory of a pediatric academic hospital. Approval for this study was granted by the hospital's institutional ethics review board. Eligible patients were prospectively recruited in the MCT clinics when a study sonographer was available. Written informed assent and consent were obtained from participants and their guardians.

\subsection{Selection of participants}

We a priori determined a pragmatic sample size of 50 patients. The referral pattern to the laboratory is one that outside physicians can request an MCT when asthma is suspected. Patients were approached in the MCT clinic if they were 6 to 17 years old. We excluded patients with any significant respiratory illness other than asthma, previous thoracic procedure, cardiac disease or anomaly, immunological diseases, severe neurodevelopmental disorders. We also excluded patients with a recent PED visit for respiratory complaints as well as patient with a history of recent viral illness (less than 4 weeks). Eligibility was determined through a combination of administered questionnaires (Appendix A,B,C) and review of the electronic medical record. At entry, $\mathrm{MCT}$ was only performed on those with a baseline $\mathrm{FEV}_{1}>70 \%$ predicted and $\mathrm{FEV}_{1} / \mathrm{FVC}$ ratio $>70 \%^{8}$.

\subsection{Methods of measurement}

Before the start of the study, all novice sonographers (AD, NM, FG) received formal LUS training from an expert sonographer (SD). An introductory course on LUS was given by the latter followed by 5 proctored 
ultrasounds. The principal investigator (SD) is certified as an independent practitioner by the Canadian Emergency Ultrasound Society and had more than 10 years of experience with point of care ultrasound.

Patients were recruited by a research assistant in the waiting room prior to their scheduled MCT appointment. Patients and parents were asked to complete the pre-test questionnaire (Appendix A), the ISAAC Asthma core Questionnaire $^{14}$ (Appendix B), and the Asthma Control Questionnaire ${ }^{21}$ (Appendix C) prior to their MCT.

Using the z.one ultra system's (Zonare, Mountain View, CA) L14-3 linear transducer, LUS was done by one of the study sonographers. A six-zone scanning protocol was performed comparable to that described by Copetti and Cattarossi ${ }^{11}$. Settings included a depth of $6-8 \mathrm{~cm}$ at a fundamental frequency (i.e. tissue harmonic imaging turned off) of $12 \mathrm{MHz}$. Ultrasound gel was layered on the probe and placed in a longitudinal manner over 6 zones (right and left anterior, mid-axillary and posterior chest zones). Six-second video clips were taken in each of the six zones.

After the first LUS, patients underwent a MCT with the dosimeter technique administered using an Aerosol Provocation System (APS) unit (MedicAid pro; CareFusion Respiratory Care) with increasing doses to match ATS recommendations ${ }^{28}$. Methacholine challenge test spirometry was conducted using a Jaeger APS spirometer (CareFusion Respiratory Care, Yorba Linda, CA). After the MCT, a second LUS was repeated in all patients. Patients who developed a $>20 \%$ fall in baseline $\mathrm{FEV}_{1}$ during the MCT were given 400 microgram salbutamol (Teva, Teva-Salbutamol HFA, Salbutamol Sulphate Inhalation Aerosol 100mcg/inh) by metered-dose inhaler after the second LUS. Patients who received salbutamol had a third LUS done shortly after (Figure 1). Sonographers were not blinded to the MCT results. Images were interpreted by the expert sonographer blinded to the patient's characteristics and MCT results. The image quality was judged adequate if the saved clips were at least 6 seconds in duration, the depth was sufficient to identify at least 2 A-lines, the gain was adequate to identify underlying structures, the images were stable enough to allow identification of lung sliding and the harmonics setting was turned off. Patients were excluded from the final analysis if one of their LUS was deemed inadequate.

\subsection{Outcome measures}

A LUS was defined as positive if there was presence of one or more findings in any of the patient's lung zones: [?]3 B-lines per intercostal space, parenchymal consolidation, pleural line abnormality, pleural effusion or absent lung sliding. A negative LUS was defined by the absence of aforementioned findings and the presence of normal lung sliding and a normal A-line pattern ${ }^{38}$. A positive MCT was defined as a decrease of $>20 \%$ of baseline $\mathrm{FEV}_{1}$ with a concentration of $8 \mathrm{mg} / \mathrm{ml}$ or less, otherwise the MCT was considered negative ${ }^{8,26}$. Asthma control was assessed via the Asthma Control Questionnaire (ACQ). We defined asthma as being poorly control if the ACQ score was above $1.5^{21}$. We used the ISSAC core questionnaire and definition to determine whether asthma was considered severe or not ${ }^{14}$.

\subsection{Primary data analysis}

Descriptive statistics were calculated for continuous and categorical variables, as medians (with IQR) and frequencies (with proportions), respectively. We conducted sub-group analysis on patients with a baseline negative LUS in order to better assess the effect of bronchospasm on ultrasound findings. We assessed associations between LUS results and patient characteristics using univariate analysis (T-tests for continuous variables and Fisher's exact test for binary variables). P-values [?]0.05 were considered significant. Multivariate logistic regression was conducted on univariate associations $\mathrm{p}<0.2$ to identify potential confounders. Data were analyzed with SPSS statistics (version 23; IBM, Armonk, NY).

\section{Results}

\subsection{Characteristics of the patients}

58 patients were approached and 44 patients were included in the final analysis (Figure 2). Clinical characteristics are presented in Table 1. 


\subsection{Main results}

At baseline, LUS was positive in 5 patients (Figure 3). All patients with a positive LUS at baseline remained positive after the MCT and salbutamol administration. 10 patients with a negative LUS at baseline converted to a positive LUS post-MCT. This group is defined as the "converters" in the discussion of this article. Out of the 9 converters that received salbutamol (ie. positive MCT), 4 patients returned to have a negative LUS post administration. Of note, 1 patient converted despite a negative MCT. Out of the 29 patients that did not convert, 16 had a post-salbutamol LUS, all of which were negative.

After the MCT, a total of 15 patients had positive LUS findings. Ten participants had [?]3 B-lines, 13 had a consolidation and 1 had a pleural anomaly. Of the 15 participants who had a positive LUS findings at any point, 8 had more than one positive finding. Fourteen of the participants had LUS findings limited to 1 or 2 intercostal spaces.

We found a significant association between a positive MCT and positive LUS findings (Odds ratio 5.3 [1.027.7] CI, $\mathrm{p}=0.05$ ) (Table 2). A sub-group analysis of the patients with a negative baseline LUS showed a non-significant, but strong correlation between a positive MCT result and positive LUS findings (Odds ratio $7.3[0.8-65.5], \mathrm{p}=0.06)$.

Univariate association identified having a short-acting beta-agonist (SABA) prescription as the only significant predictor of having a positive LUS at any point $(\mathrm{p}=0.03)$. Multivariate logistic regression also identified SABA prescription as the only significant association ( $\mathrm{p}=0.02$, OR $5.4[1.3-23.6])$.

\section{Discussion}

This is the first study to characterize LUS findings in children before and after induced bronchospasm. Eleven $\%$ of patients presenting for an MCT had positive LUS findings at baseline. Twenty-five \% of patients with a negative baseline LUS developed positive findings after the MCT. To our knowledge, this is the first study to illustrate an association between bronchospasm and LUS findings. These results contradict one of the hypotheses suggested by Dankoff et al. ${ }^{12}$ by which LUS findings found in children with asthma exacerbation could be due to viral infections. It appears that LUS findings may be due in part to a certain proportion of children with positive LUS at baseline possibly from underlying airway inflammation and in part to bronchospasm itself.

As far as we know, this is also the first report of resolution of LUS findings after the administration of salbutamol. The interpretation of this finding is limited by the small sample size, but it nonetheless supports the hypothesis that bronchospasm induces positive LUS findings that may be reversible by treating the bronchospasm ${ }^{18}$. Interestingly, salbutamol did not affect the LUS findings of patients with positive LUS at baseline. It is logical that if these positive findings are not due to bronchospasm, they also do not respond to the treatment of bronchospasm. We wonder whether serial LUS in the PED could help predict response to therapy.

Our study also challenges the notion that children with asthma should have a negative LUS as described in the adult literature ${ }^{24}$. Children may be more likely to develop airway as well as sub-segmental atelectasis. Edematous subpleural interlobular septa have been suggested as the cause for B-lines. Atelectasis could be identified as consolidation on LUS. In contrast, adult asthma may be more related to chronic airway changes and air trapping which should lead to negative LUS.

The only significant association with positive LUS identified in both univariate and multivariate analysis was being prescribed a SABA. It appears unlikely that this association is causative as salbutamol administration was actually associated with resolution of LUS findings. Therefore, it is likely that having a SABA prescription is simply a marker of being asthmatic.

A recent study by Ozkaya et al. demonstrated the utility of LUS in pediatric undifferentiated respiratory distress $^{31}$. As per their definition, a LUS "without a specific pattern" in this study combined with clinical information was strongly associated with a final diagnosis of asthma. It is important to note the critical 
difference between the definition of positive LUS in the present study and Ozkaya et al.'s definition in order to understand the apparent discrepancy between the current findings. In the current study, any positive intercostal space was interpreted as a positive LUS. Whereas Ozkaya et al. defined their findings in accordance with the definition of Volpicelli et al. of a specific pattern. For instance, a pulmonary interstitial pattern requires the presence of 2 intercostal spaces with B-lines bilaterally ${ }^{38}$. It is possible that many of the current findings would have been defined as negative by Ozkaya et al.'s protocol and many of their negative LUS would have been found positive as per the current protocol. The overwhelming majority of positive LUS findings in our study appeared small and limited to a 1 or 2 intercostal space. Since the goal of our study was to describe all LUS findings, we chose this highly sensitive definition of positivity which may not be clinically useful. Therefore, we believe that the high rate of positive LUS in the current study does not negate the utility of LUS in pediatric respiratory distress.

\section{Limitations}

There are several limitations to this study. We have opted to use the ACQ and the ISAAC scores as markers of asthma control and severity. It is important to note that these scores may not be applicable to our population as they have been validated on asthma patients only.

Novice sonographers performed the LUS. As only a representative portion of each LUS were saved for blinded assessment, sonographers were required to recognize negative LUS. It is possible that positive LUS findings have been omitted in this fashion. Prior studies done by our group ${ }^{12,37}$, showed good interrater reliability between sonographers and interpreters. Therefore, it appears unlikely that this limitation affects significantly the outcome of this study. Sonographers were not blinded to the MCT results as they needed to know whether a $3^{\text {rd }}$ LUS post-salbutamol was required or not.

LUS evaluation after salbutamol was conducted within 5 to 10 minutes after administration. Since salbutamol effect peaks around 30 minutes post-administration, there is a possibility that precocious evaluation prevented us to see more LUS finding resolution.

Since this is the first LUS study to characterize findings in children during a chemically-induced bronchospasm, our sample size was pragmatic and small. Our study is therefore not powered for sub-group analysis.

It is important to note that we evaluated LUS findings in an artificial context for convenience reasons and therefore the clinical applicability of these results must be guarded. It is unclear whether LUS findings during a chemically-induced bronchospasm are analogous to findings occurring in patient suffering from a "natural" asthma exacerbation.

\section{Conclusion}

In conclusion, This is the first known report of an association between LUS findings and bronchospasm in pediatric patients. $45 \%$ of pediatric patients who developed a chemically-induced bronchospasm during a MCT in the respiratory clinic had positive LUS findings. This is striking in contrast to what has been described in the adult literature. It is also the first documentation of resolution of LUS findings postbronchodilator administration. Most positive LUS findings were small and limited to 1 or 2 intercostal spaces. Further research is required to quantify these findings and evaluate the effect of salbutamol on LUS in the PED.

\section{Conflict of interests}

None.

\section{Author contribution statement}

ASD, AD, LL, AS, DZ conceived the study. ASD and AD supervised the conduct of the study and data collection. AD, NM and FG performed LUS. The data was managed by GA, FG, NM and ASD, including quality control. ER provided statistical advice. FG and ASD analyzed the data. FG drafted the manuscript. 


\section{Acknowledgement}

The authors acknowledge Gregory Anderson, Raphael Freitas and Joanne Ramil for their help and dedication to the project as well as Emmanouil Rampanakis for his support in statistical analysis.

\section{References}

1. Ambroggio L, Sucharew H, Rattan MS, O'Hara SM, Babcock DS, Clohessy C, Steinhoff MC, Macaluso M, Shah SS, Coley BD. 2016. Lung ultrasonography: A viable alternative to chest radiography in children with suspected pneumonia? J Pediatr. 176:93-98.e97.

2. Basile V, Di Mauro A, Scalini E, Comes P, Lofu I, Mostert M, Tafuri S, Manzionna MM. 2015. Lung ultrasound: A useful tool in diagnosis and management of bronchiolitis. BMC Pediatr. 15:63.

3. Becker A, Berube D, Chad Z, Dolovich M, Ducharme F, D'Urzo T, Ernst P, Ferguson A, Gillespie C, Kapur S et al. 2005. Canadian pediatric asthma consensus guidelines, 2003 (updated to december 2004): Introduction. Cmaj. 173(6 Suppl):S12-14.

4. Caiulo VA, Gargani L, Caiulo S, Fisicaro A, Moramarco F, Latini G, Picano E. 2011. Lung ultrasound in bronchiolitis: Comparison with chest x-ray. Eur J Pediatr. 170(11):1427-1433.

5. Caiulo VA, Gargani L, Caiulo S, Fisicaro A, Moramarco F, Latini G, Picano E, Mele G. 2013. Lung ultrasound characteristics of community-acquired pneumonia in hospitalized children. Pediatr Pulmonol. 48(3):280-287.

6. Calder A, Owens CM. 2009. Imaging of parapneumonic pleural effusions and empyema in children. Pediatr Radiol. 39(6):527-537.

7. Cattarossi L, Copetti R, Brusa G, Pintaldi S. 2016. Lung ultrasound diagnostic accuracy in neonatal pneumothorax. Can Respir J. 2016:6515069.

8. Coates AL, Wanger J, Cockcroft DW, Culver BH, Diamant Z, Gauvreau G, Hall GL, Hallstrand TS, Horvath I, de Jongh FHC et al. 2017. Ers technical standard on bronchial challenge testing: General considerations and performance of methacholine challenge tests. Eur Respir J. 49(5).

9. Coley BD. 2005. Pediatric chest ultrasound. Radiol Clin North Am. 43(2):405-418.

10. Coley BD. 2011. Chest sonography in children: Current indications, techniques, and imaging findings. Radiol Clin North Am. 49(5):825-846.

11. Copetti R, Cattarossi L. 2008. Ultrasound diagnosis of pneumonia in children. Radiol Med. 113(2):190198.

12. Dankoff S, Li P, Shapiro AJ, Varshney T, Dubrovsky AS. 2017. Point of care lung ultrasound of children with acute asthma exacerbations in the pediatric ed. Am J Emerg Med. 35(4):615-622.

13. Esposito S, Papa SS, Borzani I, Pinzani R, Giannitto C, Consonni D, Principi N. 2014. Performance of lung ultrasonography in children with community-acquired pneumonia. Ital J Pediatr. 40:37.

14. Expert panel report 3 (epr-3): Guidelines for the diagnosis and management of asthma-summary report 2007. 2007. J Allergy Clin Immunol. 120(5 Suppl):S94-138.

15. Gallard E, Redonnet JP, Bourcier JE, Deshaies D, Largeteau N, Amalric JM, Chedaddi F, Bourgeois JM, Garnier D, Geeraerts T. 2015. Diagnostic performance of cardiopulmonary ultrasound performed by the emergency physician in the management of acute dyspnea. Am J Emerg Med. 33(3):352-358.

16. Garner R, Kohen D. 2008. Changes in the prevalence of asthma among canadian children. Health Rep. 19(2):45-50.

17. Hasan AA, Makhlouf HA. 2014. B-lines: Transthoracic chest ultrasound signs useful in assessment of interstitial lung diseases. Ann Thorac Med. 9(2):99-103. 
18. Hill AB. 1965. The environment and disease: Association or causation? Proc R Soc Med. 58:295-300.

19. Ho MC, Ker CR, Hsu JH, Wu JR, Dai ZK, Chen IC. 2015. Usefulness of lung ultrasound in the diagnosis of community-acquired pneumonia in children. Pediatr Neonatol. 56(1):40-45.

20. Iuri D, De Candia A, Bazzocchi M. 2009. Evaluation of the lung in children with suspected pneumonia: Usefulness of ultrasonography. Radiol Med. 114(2):321-330.

21. Juniper EF, Gruffydd-Jones K, Ward S, Svensson K. 2010. Asthma control questionnaire in children: Validation, measurement properties, interpretation. Eur Respir J. 36(6):1410-1416.

22. Kurian J, Levin TL, Han BK, Taragin BH, Weinstein S. 2009. Comparison of ultrasound and ct in the evaluation of pneumonia complicated by parapneumonic effusion in children. AJR Am J Roentgenol. 193(6):1648-1654.

23. Lichtenstein D, Goldstein I, Mourgeon E, Cluzel P, Grenier P, Rouby JJ. 2004. Comparative diagnostic performances of auscultation, chest radiography, and lung ultrasonography in acute respiratory distress syndrome. Anesthesiology. 100(1):9-15.

24. Lichtenstein DA, Meziere GA. 2008. Relevance of lung ultrasound in the diagnosis of acute respiratory failure: The blue protocol. Chest. 134(1):117-125.

25. Loftus PA, Wise SK. 2015. Epidemiology and economic burden of asthma. Int Forum Allergy Rhinol. 5 Suppl 1:S7-10.

26. Lougheed MD, Lemiere C, Dell SD, Ducharme FM, Fitzgerald JM, Leigh R, Licskai C, Rowe BH, Bowie D, Becker A et al. 2010. Canadian thoracic society asthma management continuum-2010 consensus summary for children six years of age and over, and adults. Can Respir J. 17(1):15-24.

27. Mantuani D, Frazee BW, Fahimi J, Nagdev A. 2016. Point-of-care multi-organ ultrasound improves diagnostic accuracy in adults presenting to the emergency department with acute dyspnea. West J Emerg Med. 17(1):46-53.

28. Mazi A, Lands LC, Zielinski D. 2018. Methacholine challenge test: Comparison of tidal breathing and dosimeter methods in children. Pediatr Pulmonol. 53(2):174-180.

29. Mong A, Epelman M, Darge K. 2012. Ultrasound of the pediatric chest. Pediatr Radiol. 42(11):12871297.

30. Ortiz-Alvarez O, Mikrogianakis A. 2012. Managing the paediatric patient with an acute asthma exacerbation. Paediatr Child Health. 17(5):251-262.

31. Ozkaya AKa, Baskan Vuralkan F, Ardıc Se. 2019. Point-of-care lung ultrasound in children with non-cardiac respiratory distress or tachypnea. American Journal of Emergency Medicine. 37(11):2102-2106.

32. Pereda MA, Chavez MA, Hooper-Miele CC, Gilman RH, Steinhoff MC, Ellington LE, Gross M, Price C, Tielsch JM, Checkley W. 2015. Lung ultrasound for the diagnosis of pneumonia in children: A meta-analysis. Pediatrics. 135(4):714-722.

33. Raimondi F, Rodriguez Fanjul J, Aversa S, Chirico G, Yousef N, De Luca D, Corsini I, Dani C, Grappone L, Orfeo L et al. 2016. Lung ultrasound for diagnosing pneumothorax in the critically ill neonate. J Pediatr. 175:74-78.e71.

34. Reissig A, Copetti R. 2014. Lung ultrasound in community-acquired pneumonia and in interstitial lung diseases. Respiration. 87(3):179-189.

35. Riccabona M. 2008. Ultrasound of the chest in children (mediastinum excluded). Eur Radiol. 18(2):390399 . 
36. Samson F, Gorostiza I, Gonzalez A, Landa M, Ruiz L, Grau M. 2018. Prospective evaluation of clinical lung ultrasonography in the diagnosis of community-acquired pneumonia in a pediatric emergency department. Eur J Emerg Med. 25(1):65-70.

37. Varshney T, Mok E, Shapiro AJ, Li P, Dubrovsky AS. 2016. Point-of-care lung ultrasound in young children with respiratory tract infections and wheeze. Emerg Med J. 33(9):603-610.

38. Volpicelli G, Elbarbary M, Blaivas M, Lichtenstein DA, Mathis G, Kirkpatrick AW, Melniker L, Gargani L, Noble VE, Via G et al. 2012. International evidence-based recommendations for point-of-care lung ultrasound. Intensive Care Med. 38(4):577-591.

39. Yadav KK, Awasthi S, Parihar A. 2017. Lung ultrasound is comparable with chest roentgenogram for diagnosis of community-acquired pneumonia in hospitalised children. Indian J Pediatr. 84(7):499-504.

40. Zanobetti M, Scorpiniti M, Gigli C, Nazerian P, Vanni S, Innocenti F, Stefanone VT, Savinelli C, Coppa A, Bigiarini S et al. 2017. Point-of-care ultrasonography for evaluation of acute dyspnea in the ed. Chest. 151(6):1295-1301.

\section{Hosted file}

Tables MCT articles - pediatric pulmonology.docx available at https://authorea.com/users/ 423349/articles/528806-characterizing-pediatric-lung-ultrasound-findings-during-achemically-induced-bronchospasm 


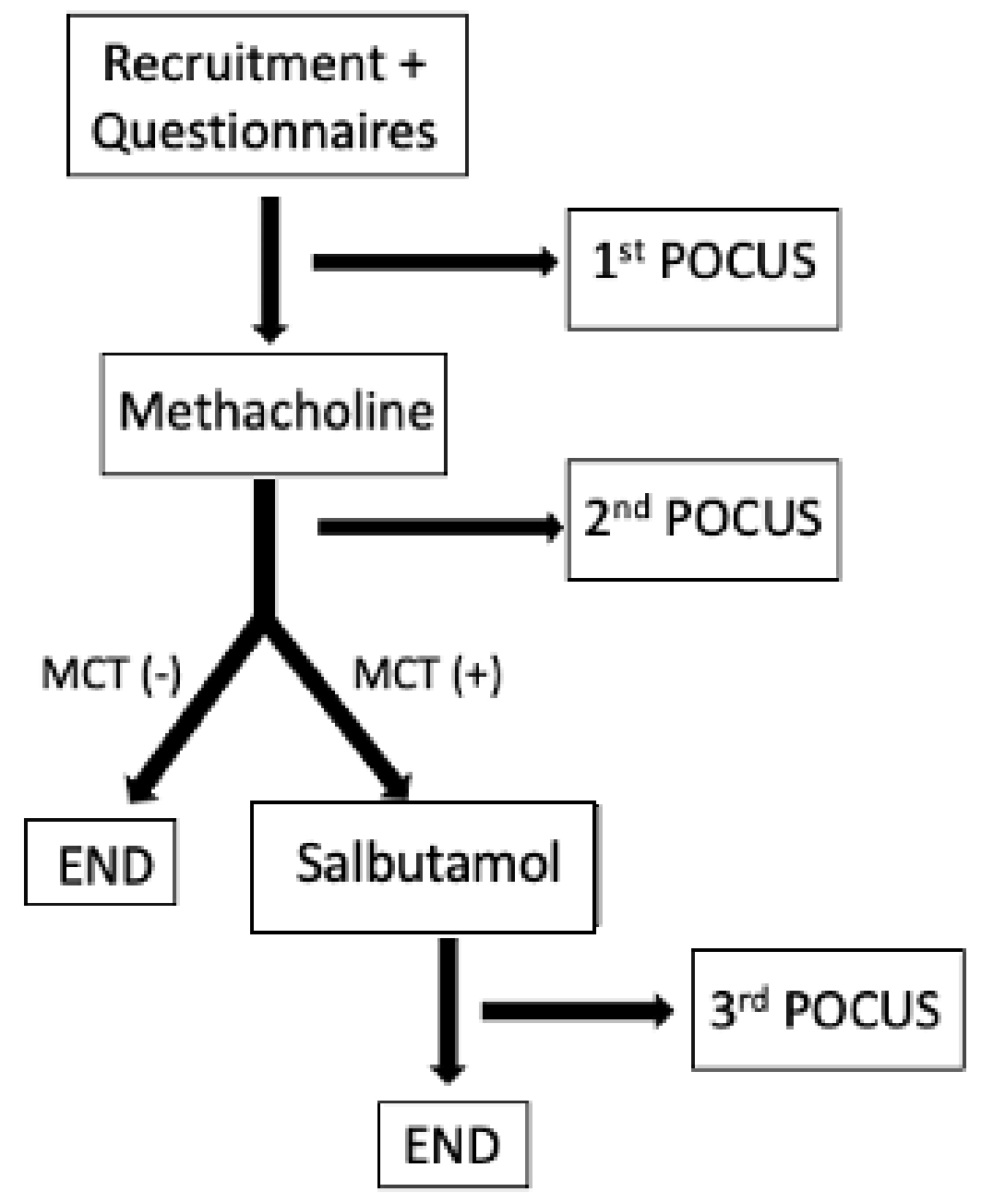




\section{Approached}

\section{Refused to participate}

57 Consented

2 Unable to complete LUS

55 Completed

5 Recent URTI

50 Included

6 Poor quality LUS

44 Analyzed 

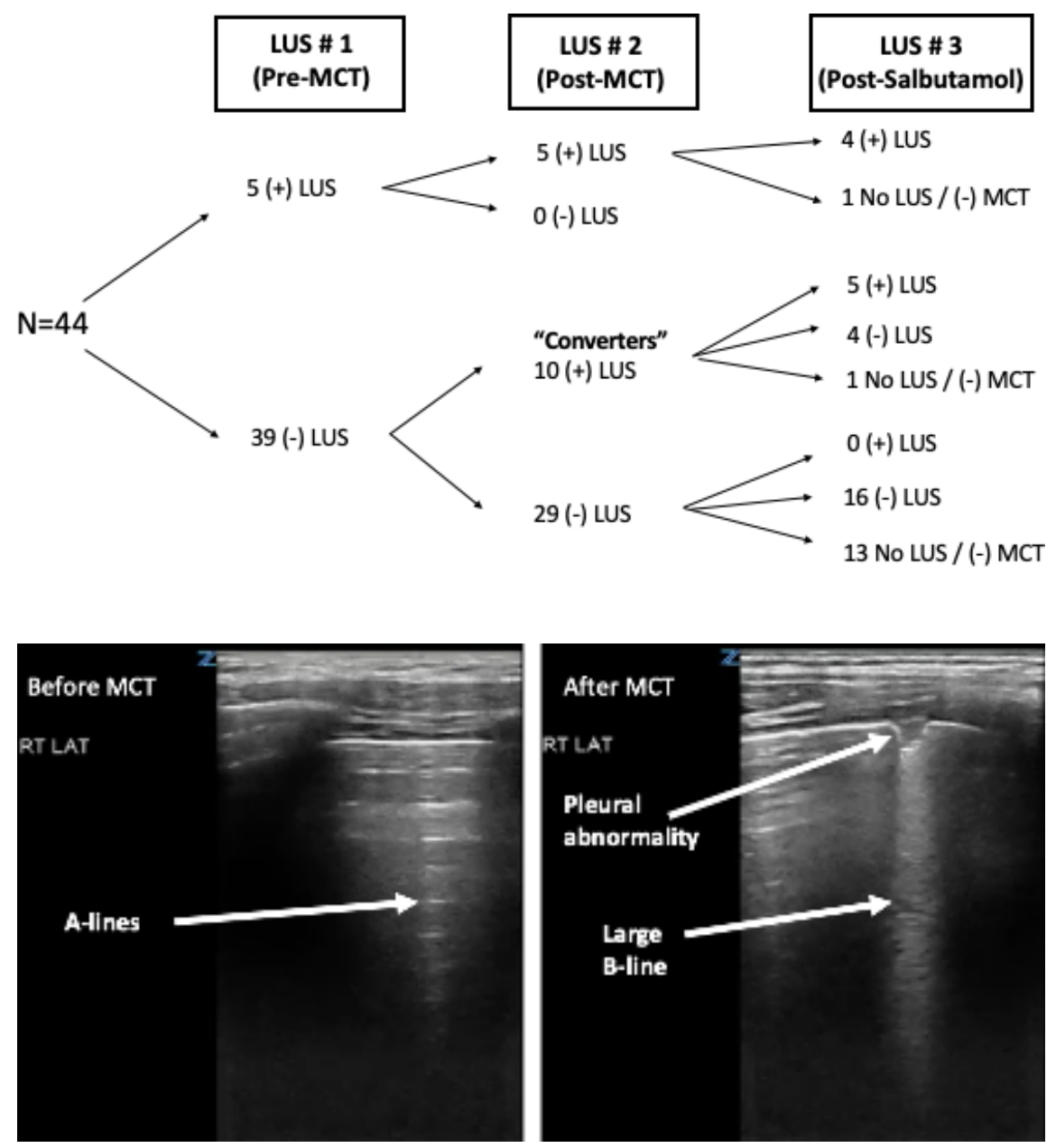